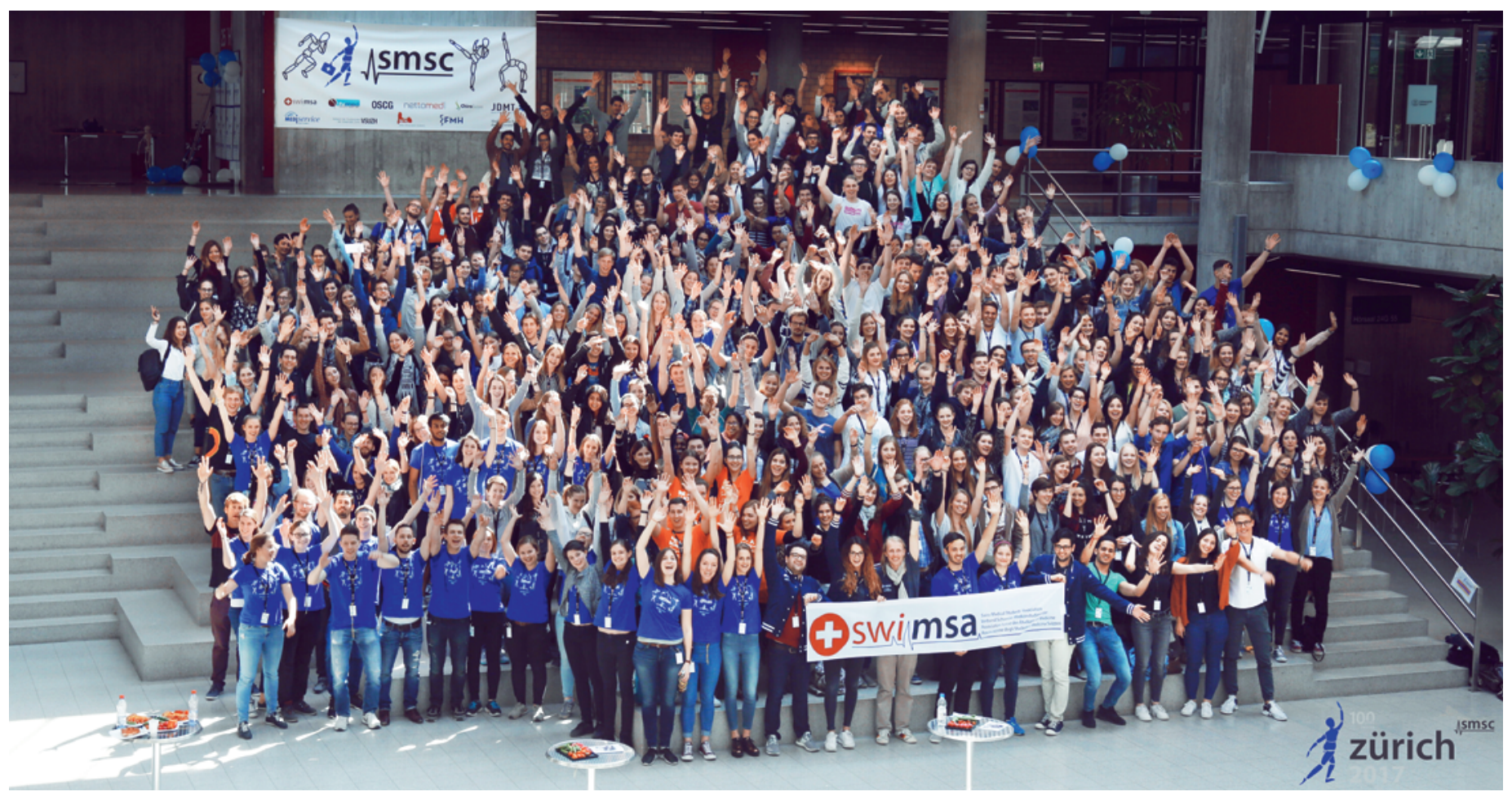

\title{
Schweizer Nachwuchs
}

\section{in Bewegung}

\section{Bea Albermann}

swimsa Liaison Officer FMH

Unter dem Thema «Let's get moving!» versammelten sich im April knapp $400 \mathrm{Me}$ dizinstudierende aus der ganzen Schweiz für die Swiss Medical Students' Convention (SMSC) in Zürich. Bereits seit 100 Jahren engagieren sich Schweizer Studierende aktiv in aussercurricularen sozialen Projekten und tragen somit einen wichtigen Teil zur Ausbildung von Ärztinnen und Ärzten bei.

Ob Aufklärung in Schulen, Unterstützung von Entwicklungsprojekten auf der anderen Seite der Welt oder Sammeln von Blutstammzellspendern - neben dem Studium engagieren sich Medizinstudierende aus der ganzen Schweiz ehrenamtlich, um als Teil der Swiss Medical Students' Association (swimsa) auf die Gesundheit der Gesellschaft aufmerksam zu machen.

So auch eine Gruppe von 20 Studierenden aus Zürich. Diese organisierte im April die Swiss Medical Students' Convention (SMSC) - einen Kongress, welcher einmal pro Semester an einer jeweils anderen Universität zu ei- nem spezifischen Thema stattfindet. Rund 350 Studierende reisten aus der ganzen Schweiz an, um an dem Wochenende mit dem diesjährigen Fokus auf Sportmedizin und Rehabilitation teilzunehmen. Dort konnten sie an in teraktiven Diskussionen, spannenden Referaten und praktischen Workshops ihren Wissensstand erweitern.

\section{Praktisches Lernen im Vordergrund}

Da das Medizinstudium vollgepackt ist mit theoretischen Vorlesungen und abstrakten Kursen, bietet sich 
den Studierenden an den SMSC-Wochenenden die Möglichkeit, das Erlernte direkt anzuwenden: Von Ultraschalldiagnostik nach Unfällen über Sport- und Essstörungen bis zu den Grundlagen der Physiotherapie und Chiropraktik - an der SMSC in Zürich wurden vielfältige Bereiche von Sportmedizin und Rehabilitation abgedeckt, welche so im normalen Vorlesungsstoff nicht behandelt werden. Thematisiert wurden auch spannende Fragen zur öffentlichen Gesundheit, beispielsweise die Rolle der physischen Aktivität in der ärztlichen Grundversorgung oder Techniken, mit welchen Hausärzte/-ärztinnen ihre Patienten zur Bewegung motivieren können.

\section{Inspiration durch Fachleute und Experten}

Doch nicht nur Studierende engagieren sich an der SMSC - die Workshops und Referate werden jeweils auf freiwilliger Basis und unentgeltlich durch spezialisierte Fachpersonen durchgeführt. Je motivierter diese von ihren Fachgebieten erzählen, desto mehr können sie die Teilnehmenden dafür begeistern und inspirieren. Weniger populäre und vertraute Bereiche der Medizin erhalten hier die attraktive Chance, den Nachwuchs unmittelbar anzusprechen und für neue Optionen zu faszinieren. Von der Entwicklung von Hightech-Prothesen an der ETH Zürich bis zur Kindersportmedizin - der Kongress präsentierte ein breites Spektrum an Berufsmöglichkeiten, mit welchen sich die Studierenden direkt auseinandersetzen und in Kontakt treten konnten. Auch das Networking untereinander als zukünftige Arbeitskollegen kam nicht zu kurz: Für die Studierenden ist die SMSC die optimale

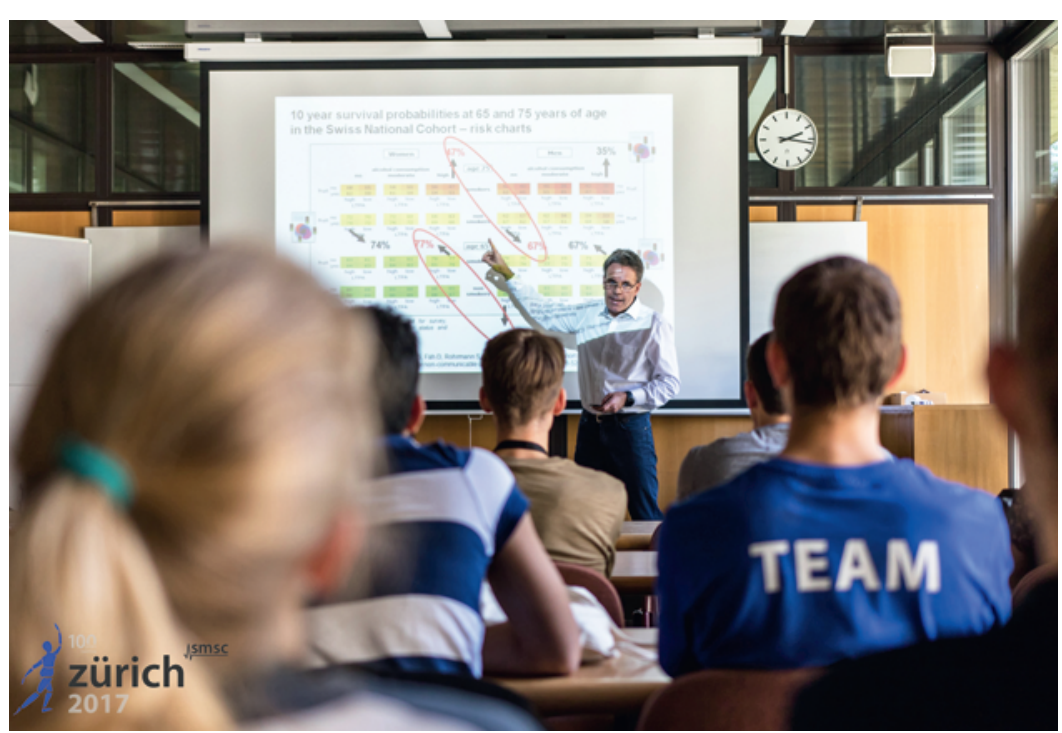

Die Workshops und Referate werden jeweils auf freiwilliger Basis und unentgeltlich von spezialisierten Fachpersonen durchgeführt.

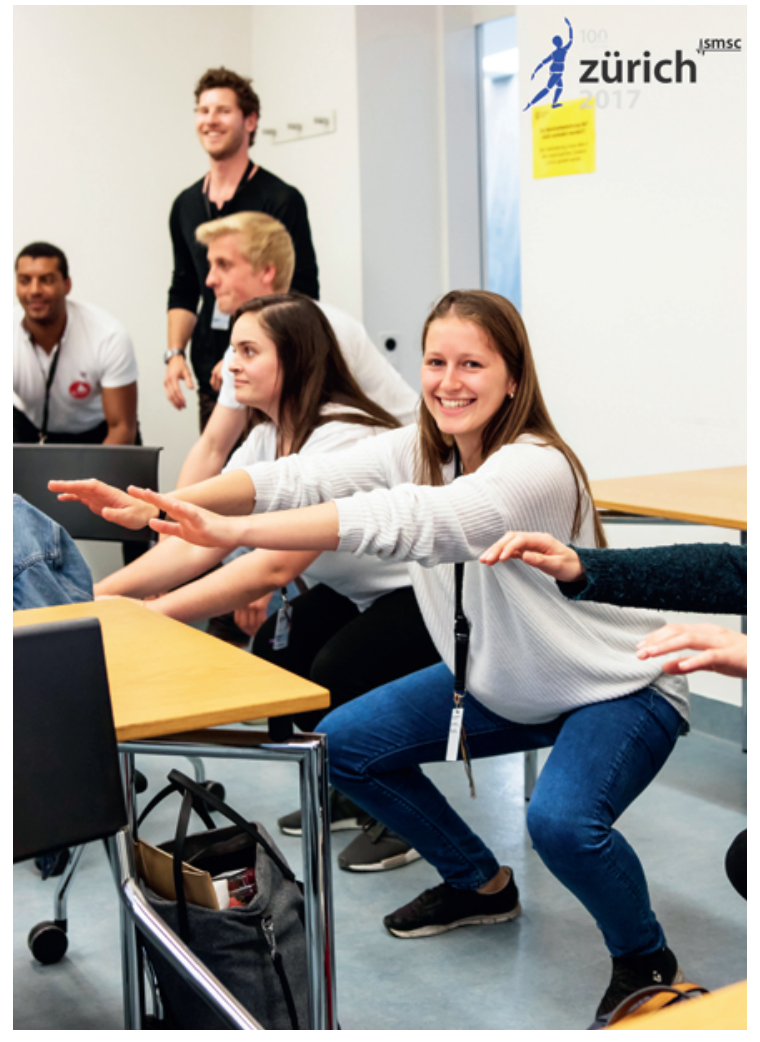

Erlerntes direkt anwenden: Am SMSC-Wochenende in Zürich haben Studierende vielfältige Bereiche von Sportmedizin und Rehabilitation vertieft, welche so im normalen Vorlesungsstoff nicht behandelt werden.

Gelegenheit, um sich auszutauschen, Projekte kennenzulernen und neue Bekanntschaften zu schliessen.

\section{Austausch und Networking sind für die Arbeit im Gesundheitswesen essentiell}

Dass dies von grundlegender Bedeutung ist, wird auch jedes Jahr an der Versammlung der International Federation of Medical Students' Association (IFMSA) deutlich - einer Art SMSC im internationalen Rahmen. Hier arbeiten Medizinstudierende aus der ganzen Welt, inklusive einer Schweizer Delegation, an innovativen Ideen und Projekten für eine nachhaltige, gesunde Gesellschaft und eine verbesserte Ausbildung von Ärzten. Dabei sind die lokalen Probleme auf den jeweiligen Kontinenten sehr unterschiedlich - von grundlegender Aufklärungsarbeit in Ghana bis zu Radiologie-Teachings in Kanada -, und somit können die verschiedenen Regionen durch Denkanstösse und Lösungsstrategien voneinander profitieren. Ohne aktiven Austausch ist dieser Fortschritt nicht denkbar. An der SMSC gelingt dies unter anderem durch eine "Project Fair», an welcher sich diverse Organisationen an Ständen vorstellen und direkt mit den Studierenden in Kontakt treten können. 


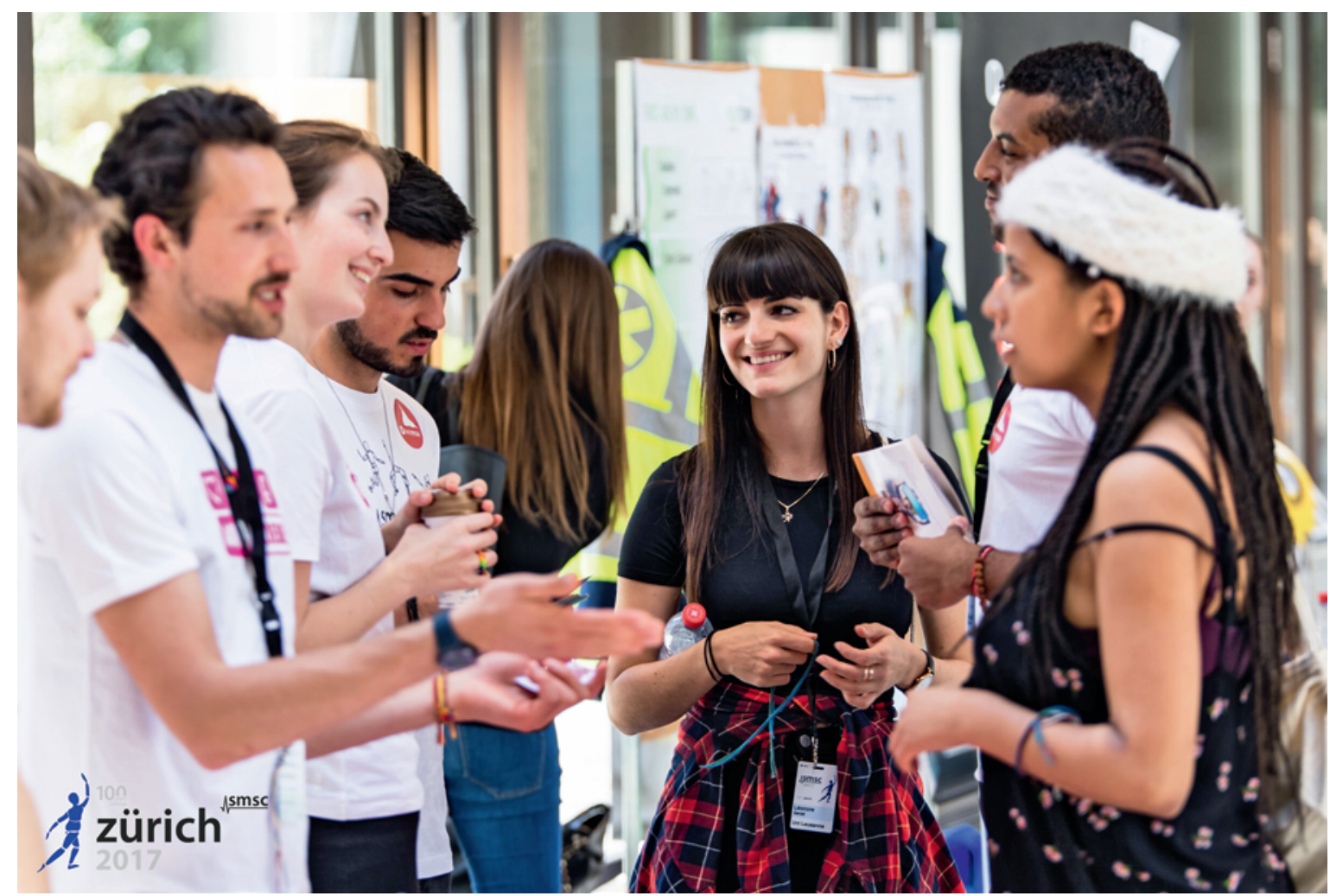

An verschiedenen Ständen können sich Studierende mit medizinischen Organisationen, potentiellen Arbeitgebern und ehrenamtlichen Projekten austauschen und neue Kontakte knüpfen.

\section{Potential bei Förderung von ausser- ordentlichem Engagement}

Die Organisation eines solch grossen Kongresses ist neben dem zeitintensiven Medizinstudium nicht selbstverständlich. Das Organisationskomitee in Zürich konnte auf die helfenden Hände von immerhin rund 50 Mitstudierenden zählen, doch waren sie auch auf die Unterstützung durch Unternehmen und professionelle Organisationen angewiesen. Um auch in Zukunft solche Anlässe zu gewährleisten, ist die swimsa somit auf stetiger Suche nach Förderung durch externe Organisationen. Und diese zahlt sich wahrlich aus: Seit exakt 100 Jahren ist die swimsa eine Inspiration für angehende Ärztinnen und Ärzte und unterstützt diese auf ihrem Weg ins Berufsleben. Das Engagement der swimsa leistet somit bei der beständig wachsenden
Anzahl an Medizinstudierenden einen entscheidenden Beitrag für die Qualität der Ärztinnen und Ärzte von morgen. Nur gemeinsam lässt sich etwas bewegen - und Bewegung hält bekanntlich gesund!

\section{Bildnachweise}

(c) swimsa

\section{Weiterführende Informationen}

Die nächste SMSC findet in Basel vom 27. bis 29. Oktober 2017 zum Thema «Forensik» statt. Weitere Infos dazu finden Sie auf unserer Website unter: www. swimsa.ch. Bei Interesse an Zusammenarbeit mit der swimsa oder allfälligen Fragen rund um die studentischen Projekte stehen wir Ihnen gerne unter folgender Adresse zur Verfügung: contact[at]swimsa.ch 